\title{
Sinais e sintomas de manifestações orais e cutâneas em crianças com COVID-19: revisão narrativa
}

\author{
Signs and symptoms of oral and skin manifestations in children with COVID-19: narrative review \\ Signos y síntomas de manifestaciones orales y cutáneas en niños con COVID-19: revisión narrativa
}

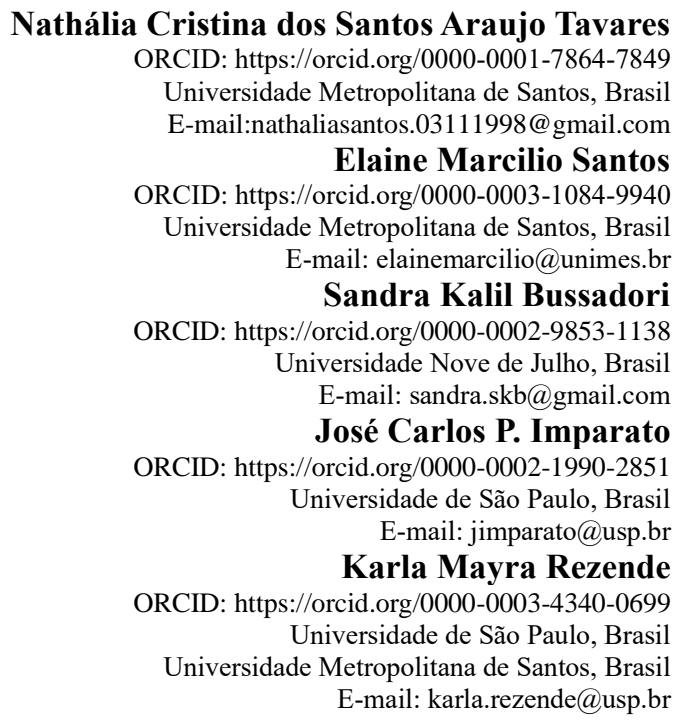

\begin{abstract}
Resumo
A pandemia da doença coronavírus 2019 é causada pela síndrome respiratória aguda grave coronavírus 2. Os sintomas clínicos mais comuns são febre, dor de cabeça, dor de garganta, dispneia, tosse seca, dor abdominal, vômito e diarreia. A relação COVID-19 e infecção de pacientes pediátricos tem algumas peculiaridades. Nos bebês, abaixo de um ano, com COVID-19 é mais frequente eles apresentarem dificuldades na alimentação e febre, já nas crianças com até nove anos, a tosse seca e a febre são mais recorrentes. Pessoas na faixa etária entre 10 e 19 anos, somam-se dores musculares, falta de ar, diarreia, falta de olfato e paladar, além de coriza. Porém, na maioria das crianças, a COVID-19 pode estar assintomática, e assim há menos conhecimento das possíveis manifestações na infância. $\mathrm{O}$ objetivo deste trabalho foi realizar uma revisão narrativa de literatura sobre as manifestações orais e cutâneas em crianças afetadas pelo COVID19 a fim de identificar lesões específicas que possam facilitar o diagnóstico e o tratamento. Metodologia: A estratégia de busca bibliográfica foi realizada nas bases de dados PubMed dos trabalhos publicados de janeiro de 2020 até 02 de agosto de 2021 utilizando os termos de pesquisa relacionados à manifestações orais e dermatológicas apresentada em crianças com COVID-19. Resultados: Foram encontrados 135 resultados, dos quais, após os critérios de inclusão, 35 foram selecionados para estudo inicial e após a eliminação dos critérios de exclusão, ficaram 26 artigos para estudo deste trabalho. Após a leitura dos textos, as manifestações bucais e dermatológicas observadas em crianças com COVID-19 foram: eritema, lesões semelhantes a frieira, língua com aparência de morango, erupções vesiculares e maculopapulares, irritação na pele, urticária, conjuntivite e síndrome multissistêmica inflamatória. Conclusão: Os sinais e sintomas em crianças com a COVID-19, apresentam algumas peculiaridades quanto comparado ao adulto. Este estudo possui algumas limitações, principalmente por abordar um assunto recente e com população restrita à pediatria. Porém, é importante para que o profissional tenha esse conhecimento e explore clinicamente e exaustivamente toda manifestação que a criança possa apresentar durante a pandemia.
\end{abstract}

Palavras-chave: Manifestações orais; Crianças; COVID-19.

\begin{abstract}
The coronavirus 2019 disease pandemic is caused by the severe acute respiratory syndrome coronavirus 2 . The most common clinical symptoms are fever, headache, sore throat, dyspnea, dry cough, abdominal pain, vomiting, and diarrhea. The relationship between COVID-19 and infection in pediatric patients has some peculiarities. In babies under one year of age, with COVID-19 it is more common to have feeding difficulties and fever, whereas in children up to nine years old, dry cough and fever are more recurrent. People who are aged between 10 and 19 years, add muscle pain, shortness of breath, diarrhea, lack of smell and taste, in addition to runny nose. However, in most children, COVID-19
\end{abstract}


may be asymptomatic, and thus there is less knowledge of the possible manifestations in childhood. The objective of this study was to carry out a narrative review of the literature on oral and cutaneous manifestations in children affected by COVID-19, in order to identify specific lesions that could facilitate diagnosis and treatment. Methodology: The bibliographic search strategy was performed in the PubMed databases of works published from January 2020 to August 2, 2021 using search terms related to oral and dermatological manifestations presented in children with COVID-19. Results: 135 results were found, of which, after the inclusion criteria, 35 were selected for the initial study and after the elimination of the exclusion criteria, there were 26 articles for the study of this work. After reading the texts, the oral and dermatological manifestations observed in children with COVID-19 were: erythema, chilblain-like lesions, strawberry-like tongue, vesicular and maculopapular eruptions, skin irritation, urticaria, conjunctivitis and multisystem inflammatory syndrome. Conclusion: The signs and symptoms in children with COVID-19 have some peculiarities when compared to adults. This study has some limitations, mainly because it addresses a recent subject and a population restricted to pediatrics. However, it is important for the professional to have this knowledge and to explore clinically and exhaustively any manifestation that the child may present during the pandemic.

Keywords: Oral manifestations; Child; COVID-19.

\begin{abstract}
Resumen
La pandemia de enfermedad del coronavirus 2019 es causada por el síndrome respiratorio agudo severo coronavirus 2. Los síntomas clínicos más comunes son fiebre, dolor de cabeza, dolor de garganta, disnea, tos seca, dolor abdominal, vómitos y diarrea. La relación entre COVID-19 e infección en pacientes pediátricos tiene algunas peculiaridades. En los bebés menores de un año, con COVID-19 es más común tener dificultades para alimentarse y fiebre, mientras que en los niños de hasta nueve años, la tos seca y la fiebre son más recurrentes. Las personas que tienen entre 10 y 19 años agregan dolor muscular, dificultad para respirar, diarrea, falta de olfato y gusto, además de secreción nasal. Sin embargo, en la mayoría de los niños, COVID-19 puede ser asintomático y, por lo tanto, hay menos conocimiento de las posibles manifestaciones en la infancia. El objetivo de este estudio fue realizar una revisión narrativa de la literatura sobre las manifestaciones orales y cutáneas en niños afectados por COVID-19, con el fin de identificar lesiones específicas que pudieran facilitar el diagnóstico y tratamiento. Metodología: La estrategia de búsqueda bibliográfica se realizó en las bases de datos PubMed de trabajos publicados desde enero de 2020 al 2 de agosto de 2021 utilizando términos de búsqueda relacionados con manifestaciones orales y dermatológicas presentadas en niños con COVID-19. Resultados: Se encontraron 135 resultados, de los cuales, luego de los criterios de inclusión, 35 fueron seleccionados para el estudio inicial y luego de la eliminación de los criterios de exclusión, hubo 26 artículos para el estudio de este trabajo. Después de la lectura de los textos, las manifestaciones orales y dermatológicas observadas en niños con COVID-19 fueron: eritema, lesiones tipo sabañones, lengua en fresa, erupciones vesiculares y maculopapulares, irritación cutánea, urticaria, conjuntivitis y síndrome inflamatorio multisistémico. Conclusión: Los signos y síntomas en niños con COVID-19 presentan algunas peculiaridades en comparación con los adultos. Este estudio tiene algunas limitaciones, principalmente porque aborda un tema reciente y una población restringida a la pediatría. Sin embargo, es importante que el profesional tenga este conocimiento y explore clínica y exhaustivamente cualquier manifestación que el niño pueda presentar durante la pandemia.
\end{abstract}

Palabras clave: Manifestaciones bucales; Niño; COVID-19.

\title{
1. Introdução
}

Nos países em desenvolvimento as doenças agudas do trato respiratório inferior constituem importante causa de internação hospitalar de crianças com idade inferior a cinco anos (Gralinski \& Baric, 2015). Na maioria das vezes, tem como etiologia viral como por exemplo o vírus respiratório sincicial, seguido do parainfluenza, influenza e adenovírus (Cruz, de Souza Luna, Alves, Conte, \& Bellei, 2021; Zhu et al., 2021).

No final do ano de 2019, na cidade de Wuhan, China, um surto desconhecido de pneumonia acometeu a população e logo se espalhou por todo mundo. As amostras de lavado broncoalveolar obtida destes pacientes identificou um betacoronavírus denominado de SARS-CoV-2. Pertence ao subgênero Sarbecovírus da família Coronaviridae e é o sétimo coronavírus conhecido a infectar seres humanos (Credie, Coelho, \& Rezende, 2020). A Covid-19 é uma infecção respiratória aguda causada pelo coronavírus SARS-CoV-2, potencialmente grave, de elevada transmissibilidade e de distribuição global (Baggio et al., 2021).

A transmissão do SARS-CoV-2 ocorre por gotículas de saliva, especialmente quando o indivíduo infectado tosse ou espirra. Assim, o contato com objetos e mãos contaminados com as mucosas constituem importantes portas de entrada para o vírus. Ademais, é possível que ocorra transmissão via fecal-oral, já que foi constatado em estudo a presença do vírus no reto, mesmo após exames da nasofaringe resultarem em negativo (To et al., 2020). 
Ao contrário dos adultos, as crianças com a COVID-19 apresentam sintomas clínicos mais brandos, e em muitos casos, assintomáticos. Porém, mesmo nestas condições não impede de transmitir o vírus (Mehta et al., 2020; Santos et al., 2020).

A aplicação da vacina iniciou após um ano de pandemia, ou seja, em janeiro de 2021 começando nas populações mais idosas e adultos. E, apesar dos dados promissores de segurança e imunogenicidade em adultos, até fevereiro de 2021, os ensaios clínicos em crianças ainda não tinham sido iniciados nos Estados Unidos (Kamidani, Rostad, \& Anderson, 2021). Portanto, o espectro completo de apresentações clinicas em crianças ainda precisa ser observado e descrito. A mucosa oral é talvez, o tecido com maior probabilidade de ser comprometido por doenças sistemáticas e adquirida. Assim, o objetivo deste artigo foi identificar, por meio de uma revisão narrativa de literatura, as principais manifestações bucais e dermatológicas que, crianças com a COVID19 , podem apresentar.

\section{Metodologia}

Uma busca eletrônica foi realizada na base de dados Pubmed/MedLine entre 01 de janeiro de 2020 até 02 de agosto de 2021, para os artigos em inglês. No modo "pesquisa avançada, foram utilizados os termos (MeSH) e os Entry Terms combinados com os termos booleanos "OR"e "AND".

Pubmed: ((((Covid-19 skin) OR (Fernandez-Nieto et al.)) OR (Fernandez-Nieto et al.)) OR (Aghazadeh, Homayouni, \& Sartori-Valinotti) AND ((review[Filter] OR systematicreview[Filter]) AND ((Child) OR (Adelson \& Goldfried))

Após a busca, foi realizada a triagem e coleta de dados. Os títulos e resumos foram verificados quanto aos critérios de inclusão: estudos publicados na língua inglesa, patologias bucais e dermatológicas e em crianças. Em seguida, os textos completos foram estudados. Foram excluídos artigos fora da faixa pediátrica e aqueles que não estavam relacionados ao tema. Para os estudos, os dados coletados de cada artigo incluído foram: ano, autores, título, tipo de doenças. Os dados foram analisados de maneira descritiva.

\section{Resultados}

Na busca para realização narrativa de literatura, foram identificados 135 títulos no Pubmed no período de janeiro de 2020 até 02 de agosto de 2021. Destes, 100 foram excluídos por estarem fora do tema (como adultos e ou manifestações virais não relacionados ao COVID-19). Os 35 artigos que restaram, após a leitura do resumo, 10 foram excluídos porque não os manuscritos não mencionavam a idade.

As principais informações contidas em cada artigo encontram-se descritas no Quadro 1. 


\section{Quadro 1 - Consolidação da revisão narrativa.}

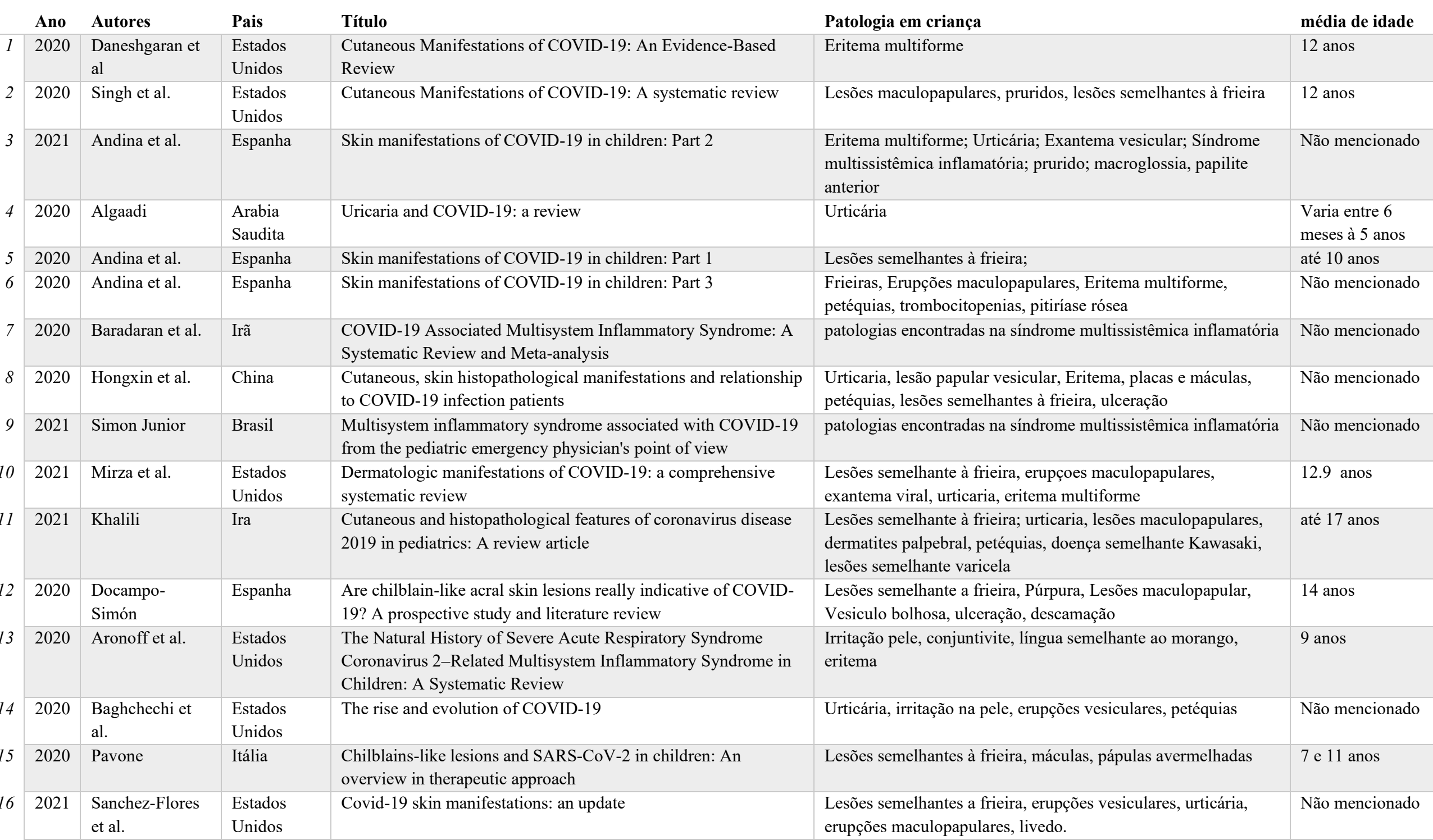


Research, Society and Development, v. 10, n. 10, e258101018515, 2021

(CC BY 4.0) | ISSN 2525-3409 | DOI: http://dx.doi.org/10.33448/rsd-v10i10.18515

\begin{tabular}{|c|c|c|c|c|c|c|}
\hline 17 & 2021 & Halepas et al. & $\begin{array}{l}\text { Estados } \\
\text { Unidos }\end{array}$ & $\begin{array}{l}\text { Oral manifestations of COVID-2019-related multisystem } \\
\text { inflammatory syndrome in children: a review of } 47 \text { pediatric } \\
\text { patients }\end{array}$ & Conjuntivite, língua em morango, lábios rachados, eritema & 9 anos \\
\hline 18 & 2021 & Toraih et al. & $\begin{array}{l}\text { Estados } \\
\text { Unidos }\end{array}$ & $\begin{array}{l}\text { Multisystem inflammatory syndrome in pediatric COVID-19 } \\
\text { patients: a meta-analysis }\end{array}$ & Síndrome multissistemica inflamatória, maculopapular, & 9.10 anos \\
\hline 19 & 2021 & Naka et al. & $\begin{array}{l}\text { Estados } \\
\text { Unidos }\end{array}$ & $\begin{array}{l}\text { A dermatologic perspective on multisystem inflammatory } \\
\text { syndrome in children }\end{array}$ & $\begin{array}{l}\text { Lesões semelhantes a frieira, erupções maculopapulares, } \\
\text { urticária, lesões tipo varicela, eritema multiforme }\end{array}$ & Não mencionado \\
\hline 20 & 2021 & $\begin{array}{l}\text { Larenas- } \\
\text { Linnemann et al. }\end{array}$ & México & $\begin{array}{l}\text { Cutaneous Manifestations Related to COVID-19 Immune } \\
\text { Dysregulation in the Pediatric Age Group }\end{array}$ & $\begin{array}{l}\text { Eritema multiforme, lesão maculopapular, irritação na pele, } \\
\text { edema, urticária, lesões vesiculares, Síndrome multissistemica } \\
\text { inflamatoria }\end{array}$ & Não mencionado \\
\hline 21 & 2021 & Shah et al. & India & $\begin{array}{l}\text { Cutaneous manifestations associated with COVID-19 in children: } \\
\text { A systematic review }\end{array}$ & $\begin{array}{l}\text { Eritema multiforme, máculas, placas, prurido, lesão reticular, } \\
\text { exantema viral, }\end{array}$ & Até 18 anos \\
\hline 22 & 2021 & Farooq et al. & Paquistão & $\begin{array}{l}\text { Multisystem Inflammatory Syndrome in Children and } \\
\text { Adolescents (MIS-C) under the Setting of COVID-19: A Review } \\
\text { of Clinical Presentation, Workup and Management }\end{array}$ & $\begin{array}{l}\text { Lesões eritematosas, lesões semelhantes à frieira, síndrome } \\
\text { multissistemica inflamatória }\end{array}$ & \\
\hline 23 & 2021 & Marzano et al. & Itália & $\begin{array}{l}\text { Multisystem Inflammatory Syndrome in Children Associated } \\
\text { with COVID-19: A Review with an Emphasis on Mucocutaneous } \\
\text { and Kawasaki Disease-Like Findings. }\end{array}$ & $\begin{array}{l}\text { Erupção cutâneas, conjuntivite, lábios ressecados, edemas nas } \\
\text { mãos e pés, língua em formato de morango, descamação na } \\
\text { pele, síndrome multissistemica inflamatória }\end{array}$ & Não mencionado \\
\hline 24 & 2021 & Lacina et al. & $\begin{array}{l}\text { Republica } \\
\text { Tcheca }\end{array}$ & $\begin{array}{l}\text { Pediatric Inflammatory Multisystem Syndrome (PIMS) - } \\
\text { Potential Role for Cytokines Such Is IL-6 }\end{array}$ & Síndrome multissistêmica inflamatória & Não mencionado \\
\hline 25 & 2021 & Mercier et al. & França & $\begin{array}{l}\text { Severe acute respiratory syndrome coronavirus 2-related } \\
\text { multisystem inflammatory syndrome in children mimicking } \\
\text { Kawasaki disease. }\end{array}$ & Síndrome multissistêmica inflamatória & Não mencionado \\
\hline
\end{tabular}

Fonte: Autores. 


\section{Discussão}

Os trabalhos estudados para essa pesquisa, têm demonstrado que as crianças infectadas pelo COVID-19 irão apresentar algum tipo de lesão estomatológica e ou dermatológica, que auxiliarão na identificação da doença, principalmente naquelas que estão assintomáticas. A etiologia da infecção pelo Sars em crianças, em $36 \%$ dos casos, foi porque elas estiveram em contato próximos com um adulto infectado, principalmente em ambiente residencial ou de saúde (Cheng et al., 2005). Isso porque a principal via de transmissão, consistente com o coronavirus e outros vírus respiratórios comuns, como a gripe, é por meio de gotículas respiratórias que são geradas ao falar, tossir, ou espirrar quando uma pessoa infectada (Credie et al., 2020)'(Anfinrud, Stadnytskyi, Bax, \& Bax, 2020). Quando estudamos sobre os sintomas que a COVID-19 possa apresentar em crianças e adolescentes, incluem sintomas leves, moderados, graves e críticos. No entanto, há evidências crescentes que muitas infecções são assintomáticas, no qual a criança pode estar clinicamente bem e mesmo assim transmitir o vírus para outras pessoas (Gao et al., 2021; Kronbichler et al., 2020). Já em adultos, as observações clinicas mais frequentes são: tosse, febre, falta de ar, mialgia, fadiga e dor de cabeça (Aghazadeh et al., 2020; Huang et al., 2020; Rajapakse \& Dixit, 2021). Em 2021, Mantovani et al., publicaram uma revisão sistemática com meta análise sobre os sinais clínicos que crianças e adolescentes podem apresentar. Dos 19 estudos incluídos com 2855 crianças e adolescentes, menos da metade (47\%) apresentaram febre, congestão nasal, dispneia, dor abdominal (Mantovani et al., 2021). Isso demonstra que as crianças tem uma boa evolução de cura, porém é extremamente necessário de cuidados gerais e isolamento social por 14 dias para evitar transmitir para outras pessoas. Além disso, a sobreposição com sintomas de outras infecções virais do trato respiratório comumente observadas nos casos de influenza por exemplo, impõe desafios adicionais para o reconhecimento da COVID-19. Uma possível forma de consideração desta doença pode estar relacionada com as lesões orais e dermatológicas. Isso porque a apresentação clínica geral na criança diferem daqueles em adultos. Erupções vesiculares, eritema multiforme, pruridos e urticárias foram os próximos achados dermatológicos mais frequentes em quaisquer pessoas, porém, a síndrome multissistêmica inflamatória foram observadas apenas em crianças e adolescentes (Andina et al., 2021). Lesões vesiculares, no entanto, tendem a ser mais específicas para exantemas virais. Como tal, as lesões vesiculares podem ser uma ferramenta de diagnóstico COVID-19 mais útil (Andina et al., 2021).

Já na região perioral, podem estar presentes vesículas e erosões envolvendo lábios, língua e mucosa bucal. Outras lesões mais comumentes encontradas na pele foram as petéquias, que também podem estar localizadas no lábio inferior, palato e mucosa da orofaringe, dorso da língua, gengivas e palatos (Corchuelo \& Ulloa, 2020). As dermatites palpebrais e conjuntivite também foram observadas em crianças com a COVID-19 (Khalili, Iranmanesh, Mohammadi, \& Aflatoonian, 2021; Marzano et al., 2021).

Uma alteração observada nos 26 estudos analisados, foi a presença do exantema viral nos estágios iniciais da doença COVID-19 em crianças e, ocasionalmente, mesmo antes do início de outras manifestações (Galvan Casas et al., 2020; Li, Zhao, Zhou, \& Hu, 2020). Outra lesão interessante e presente nos casos observados em criança, foi aquelas semelhantes às frieiras no pés. Essas lesões parecem afetar principalmente os pés de crianças e adolescentes com boa saúde sistêmica sem histórico de doenças recentes ou crônicas. Vários estudos, de diversos países, relatam sintomas consistentes com infecção por SARS-CoV2 nos dias e semanas anteriores ao aparecimento de frieiras ou contato com pessoas que apresentaram esses sintomas (Andina et al., 2021; Docampo-Simon et al., 2020; Khalili et al., 2021; Li et al., 2020; Singh, Kaur, Singh, \& Sen, 2021). Foi também relatado nos artigos, que a exposição de crianças ao SARS-CoV-2 resulta no desenvolvimento da Síndrome Inflamatória Multissistêmica (Kabeerdoss et al., 2021; Santos et al., 2020). O diagnóstico é baseado em critérios clínicos que incluem febre por pelo menos cinco dias e presença de algumas características clinicas como: injeção conjuntival bilateral; linfadenopatia cervical; alterações da mucosa oral; erupções cutâneas polimórficas; edema ou vermelhidão das extremidades e exclusão de diagnósticos alternativos. A febre pode fazer com que a criança atinja picos de temperatura frequentemente excedendo $39^{\circ} \mathrm{C}$ ou mais e quando não tratadas, o período febril dura em média 11 dias (Farooq et al., 2021; Kabeerdoss et al., 2021). 
No estudo de coorte de Halepas et al., 2021(Halepas et al., 2021), após acompanhar 47 crianças com idade média de 9 anos de idade e que foram diagnosticada com Covid-19, a presença de lábios avermelhados e inchados e língua com aparência de morango. Alías, a língua com aparência de morango (ou língua de framboesa) é um exantema distinto do dorso da língua caracterizado pela proeminência de papilas fungiformes hipertróficas e inflamadas juntamente com hiperemia. Embora seja uma característica diagnóstica de distúrbios como a doença de Kawasaki e escarlatina, foi também observada em crianças com Covid19 (Rekhtman et al., 2021; Sert, 2021).

As manifestações cutâneas do COVID-19 podem ajudar na identificação de portadores, permitindo que os profissionais de saúde implementem medidas apropriadas para conter a disseminação e, se apropriado, forneçam cuidados específicos do COVID-19 a esses indivíduos.

\section{Conclusão}

Essa revisão narrativa de literatura, se propôs a descrever quais são as peculiaridades sobre os sinais e sintomas das manifestações orais e cutâneas do Sars-CoV-2 na classe pediátrica por meio de análise e interpretação das pesquisas já existentes, buscando reunir e sintetizar conhecimentos sobre o tema para os profissionais de saúde e professores com objetivo de estarem atentos para possíveis apresentação atípicas em crianças.

\section{Referências}

Adelson, R., \& Goldfried, M. R. (1970). Modeling and the fearful child patient. ASDC J Dent Child, 37(6), 476 passim. Retrieved from https://www.ncbi.nlm.nih.gov/pubmed/4249222

Aghazadeh, N., Homayouni, M., \& Sartori-Valinotti, J. C. (2020). Oral vesicles and acral erythema: report of a cutaneous manifestation of COVID-19. Int J Dermatol, 59(9), 1153-1154. doi:10.1111/ijd.15047

Andina, D., Belloni-Fortina, A., Bodemer, C., Bonifazi, E., Chiriac, A., Colmenero, I., \& COVID, E. G. f. t. S. M. o. (2021). Skin manifestations of COVID-19 in children: Part 1. Clin Exp Dermatol, 46(3), 444-450. doi:10.1111/ced.14481

Anfinrud, P., Stadnytskyi, V., Bax, C. E., \& Bax, A. (2020). Visualizing Speech-Generated Oral Fluid Droplets with Laser Light Scattering. N Engl J Med, 382(21), 2061-2063. doi:10.1056/NEJMc2007800

Baggio, S., L'Huillier, A. G., Yerly, S., Bellon, M., Wagner, N., Rohr, M., \& Eckerle, I. (2021). Severe Acute Respiratory Syndrome Coronavirus 2 (SARS-CoV-2) Viral Load in the Upper Respiratory Tract of Children and Adults With Early Acute Coronavirus Disease 2019 (COVID-19). Clin Infect Dis, 73(1), 148-150. doi:10.1093/cid/ciaa1157

Cheng, F. W., Ng, P. C., Chiu, W. K., Chu, W. C., Li, A. M., Lo, K. L., \& Fok, T. F. (2005). A case-control study of SARS versus community acquired pneumonia. Arch Dis Child, 90(7), 747-749. doi:10.1136/adc.2004.063446

Corchuelo, J., \& Ulloa, F. C. (2020). Oral manifestations in a patient with a history of asymptomatic COVID-19: Case report. Int J Infect Dis, 100, 154-157. doi:10.1016/j.ijid.2020.08.071

Credie, G. B., Coelho, A. A., \& Rezende, K. M. (2020). Coronavirus (COVID-19) in children: history and pediatric oral health. Dental Oral biology and craniofacial research, 3(3), 3. doi:http://dx.doi.org/10.31487/j.DOBCR.2020.03.06

Cruz, J. S., de Souza Luna, L. K., Alves, V. R. G., Conte, D. D., \& Bellei, N. C. J. (2021). Viral load of respiratory syncytial virus among children from primary care and hospital settings admitted to a university hospital in Brazil (2009-2013). $J$ Med Virol, 93(6), 3397-3400. doi:10.1002/jmv.26185

Docampo-Simon, A., Sanchez-Pujol, M. J., Juan-Carpena, G., Palazon-Cabanes, J. C., Vergara-De Caso, E., Berbegal, L., \& Betlloch-Mas, I. (2020). Are chilblain-like acral skin lesions really indicative of COVID-19? A prospective study and literature review. J Eur Acad Dermatol Venereol, 34(9), e445-e447. doi:10.1111/jdv.16665 
Farooq, A., Alam, F., Saeed, A., Butt, F., Khaliq, M. A., Malik, A., \& Abdullah, M. (2021). Multisystem Inflammatory Syndrome in Children and Adolescents (MIS-C) under the Setting of COVID-19: A Review of Clinical Presentation, Workup and Management. Infect Dis (Auckl), 14, 11786337211026642 . doi:10.1177/11786337211026642

Fernandez-Nieto, D., Ortega-Quijano, D., Jimenez-Cauhe, J., Burgos-Blasco, P., de Perosanz-Lobo, D., Suarez-Valle, A., . . . Fernandez-Guarino, M. (2020). Clinical and histological characterization of vesicular COVID-19 rashes: a prospective study in a tertiary care hospital. Clin Exp Dermatol, 45(7), 872-875. doi:10.1111/ced.14277

Galvan Casas, C., Catala, A., Carretero Hernandez, G., Rodriguez-Jimenez, P., Fernandez-Nieto, D., Rodriguez-Villa Lario, A., . . Garcia-Doval, I. (2020). Classification of the cutaneous manifestations of COVID-19: a rapid prospective nationwide consensus study in Spain with 375 cases. Br J Dermatol, 183(1), 71-77. doi:10.1111/bjd.19163

Gao, Z., Xu, Y., Sun, C., Wang, X., Guo, Y., Qiu, S., \& Ma, K. (2021). A systematic review of asymptomatic infections with COVID-19. J Microbiol Immunol Infect, 54(1), 12-16. doi:10.1016/j.jmii.2020.05.001

Gralinski, L. E., \& Baric, R. S. (2015). Molecular pathology of emerging coronavirus infections. J Pathol, $235(2), 185-195$. doi:10.1002/path.4454

Halepas, S., Lee, K. C., Myers, A., Yoon, R. K., Chung, W., \& Peters, S. M. (2021). Oral manifestations of COVID-2019-related multisystem inflammatory syndrome in children: a review of 47 pediatric patients. J Am Dent Assoc, 152(3), $202-208$. doi:10.1016/j.adaj.2020.11.014

Huang, C., Wang, Y., Li, X., Ren, L., Zhao, J., Hu, Y., \& Cao, B. (2020). Clinical features of patients infected with 2019 novel coronavirus in Wuhan, China. Lancet, 395(10223), 497-506. doi:10.1016/S0140-6736(20)30183-5

Kabeerdoss, J., Pilania, R. K., Karkhele, R., Kumar, T. S., Danda, D., \& Singh, S. (2021). Severe COVID-19, multisystem inflammatory syndrome in children, and Kawasaki disease: immunological mechanisms, clinical manifestations and management. Rheumatol Int, 41(1), 19-32. doi:10.1007/s00296-020-04749-4

Kamidani, S., Rostad, C. A., \& Anderson, E. J. (2021). COVID-19 vaccine development: a pediatric perspective. Curr Opin Pediatr, 33(1), 144-151. doi:10.1097/MOP.0000000000000978

Khalili, M., Iranmanesh, B., Mohammadi, S., \& Aflatoonian, M. (2021). Cutaneous and histopathological features of coronavirus disease 2019 in pediatrics: A review article. Dermatol Ther, 34(1), e14554. doi:10.1111/dth.14554

Kronbichler, A., Kresse, D., Yoon, S., Lee, K. H., Effenberger, M., \& Shin, J. I. (2020). Asymptomatic patients as a source of COVID-19 infections: A systematic review and meta-analysis. Int J Infect Dis, 98, 180-186. doi:10.1016/j.ijid.2020.06.052

Li, H., Zhao, Y., Zhou, L., \& Hu, J. (2020). Cutaneous, skin histopathological manifestations and relationship to COVID-19 infection patients. Dermatol Ther, 33(6), e14157. doi:10.1111/dth.14157

Mantovani, A., Rinaldi, E., Zusi, C., Beatrice, G., Saccomani, M. D., \& Dalbeni, A. (2021). Coronavirus disease 2019 (COVID19 ) in children and/or adolescents: a meta-analysis. Pediatr Res, 89(4), 733-737. doi:10.1038/s41390-020-1015-2

Marzano, A. V., Cassano, N., Moltrasio, C., Verdoni, L., Genovese, G., \& Vena, G. A. (2021). Multisystem Inflammatory Syndrome in Children Associated with COVID-19: A Review with an Emphasis on Mucocutaneous and Kawasaki Disease-Like Findings. Dermatology, 1-9. doi:10.1159/000515449

Mehta, N. S., Mytton, O. T., Mullins, E. W. S., Fowler, T. A., Falconer, C. L., Murphy, O. B., \& Nguyen-Van-Tam, J. S. (2020). SARS-CoV-2 (COVID-19): What Do We Know About Children? A Systematic Review. Clin Infect Dis, 71(9), $2469-2479$. doi: $10.1093 / \mathrm{cid} / \mathrm{ciaa} 556$

Rajapakse, N., \& Dixit, D. (2021). Human and novel coronavirus infections in children: a review. Paediatr Int Child Health, 41(1), 36-55. doi:10.1080/20469047.2020.1781356

Rekhtman, S., Tannenbaum, R., Strunk, A., Birabaharan, M., Wright, S., \& Garg, A. (2021). Mucocutaneous disease and related clinical characteristics in hospitalized children and adolescents with COVID-19 and multisystem inflammatory syndrome in children. J Am Acad Dermatol, 84(2), 408-414. doi:10.1016/j.jaad.2020.10.060 
Santos, C. N., Rezende, K. M., Oliveira Neto, N. F., Okay, T. S., Braz-Silva, P. H., \& Bonecker, M. (2020). Saliva: an important alternative for screening and monitoring of COVID-19 in children. Braz Oral Res, 34, e0125. doi:10.1590/1807-3107bor2020.vol34.0125

Sert, A. (2021). A response to "Mucocutaneous disease and related clinical characteristics in hospitalized children and adolescents with COVID-19 and multisystem inflammatory syndrome in children". J Am Acad Dermatol, 84(6), e301-e302. doi:10.1016/j.jaad.2021.02.076

Singh, H., Kaur, H., Singh, K., \& Sen, C. K. (2021). Cutaneous Manifestations of COVID-19: A Systematic Review. Adv Wound Care (New Rochelle), 10(2), 51-80. doi:10.1089/wound.2020.1309

To, K. K., Tsang, O. T., Yip, C. C., Chan, K. H., Wu, T. C., Chan, J. M., \& Yuen, K. Y. (2020). Consistent Detection of 2019 Novel Coronavirus in Saliva. Clin Infect Dis, 71(15), 841-843. doi:10.1093/cid/ciaa149

Zhu, G., Xu, D., Zhang, Y., Wang, T., Zhang, L., Gu, W., \& Shen, M. (2021). Epidemiological characteristics of four common respiratory viral infections in children. Virol J, 18(1), 10. doi:10.1186/s12985-020-01475-y 